\title{
PONTO DE EQUILÍBRIO DO MANEJO DE BAIXO IMPACTO EM FLORESTAS TROPICAIS - UM ESTUDO DE CASO
}

\author{
Romano Timofeiczyk Júnior ${ }^{1}$, Ricardo Berger ${ }^{1}$, Versides Sebastião Moraes e Silva ${ }^{2}$, \\ Roberto Antonio Ticle de Melo e Sousa ${ }^{2}$ \\ ${ }^{1}$ Eng. Florestal, Dr., Depto. de Economia Rural e Extensão, UFPR, Curitiba, PR, Brasil - romano@ufpr.br - berger@bighost.com.br \\ ${ }^{2}$ Eng. Florestal, Dr., Faculdade de Engenharia Florestal da UFMT, Cuiaba, Mt, Brasil - versides@uol.com.br - ratms@ terra.com.br \\ Recebido para publicação: 19/02/2008 - Aceito para publicação: 12/08/2008
}

\begin{abstract}
Resumo
Este artigo teve como objetivo determinar o ponto de equilíbrio monetário e de área relacionado com o manejo de florestas tropicais em escala empresarial e com dois ciclos de corte. Os dados utilizados foram fornecidos por uma empresa que explora uma floresta sob regime de manejo sustentado, situada no Mato Grosso, e que comercializa as toras com as indústrias da região. Com base nas informações, foram determinados os custos fixos, os custos variáveis e as receitas provenientes das várias espécies comercializadas, e calculado o ponto de equilíbrio de área e monetário, com e sem custo de oportunidade da terra. Os resultados demonstraram que o manejo florestal requer uma extensa área de terra para que ocorra sustentabilidade econômica. Considerando o custo de oportunidade da terra, verificou-se um incremento significativo no tamanho da área necessária para que haja igualdade entre custos e receitas.

Palavras-chave: Manejo florestal; ponto de equilíbrio; estrutura de custos; custo da terra.
\end{abstract}

\begin{abstract}
Break-even-point of the tropical forest management - a case study. This paper aimed to determine the area and the monetary break-even-point related to the management of rainforests, in business scale, with two cycles of harvesting. The studied data were provided by a company which harvests a rainforest under the scheme of sustainable management, located in Mato Grosso State, Brazil. This company markets logs with the local industries. There were determined the fixed and variable costs and the receipts of the different trade species. After, it was calculated the area and monetary breakeven-point, with and without the land opportunity cost. The results showed that the rainforest management demands large areas in order to have economic sustainability. Considering the land opportunity cost, it was noticed a significant increase of the area demanded in order to have equality between costs and receipts.

Keywords: Forest management; break-even-point; cost framework; land costs.
\end{abstract}

\section{INTRODUÇÃO}

A Amazônia, em função da sua biodiversidade, da importância na regulação do clima e na proteção de mananciais e dos solos, além de fornecedora de produtos madeireiros e não-madeireiros, é uma das mais relevantes regiões fitogeográficas do mundo. A utilização racional da floresta amazônica significa o equilíbrio de vários fatores e funções que garantem o funcionamento de seu conjunto de ecossistemas, fornecendo benefícios importantes na forma de serviços ambientais que são frequentemente necessários para a sustentabilidade econômica (HIGUCHI, 1994). Em razão da sua importância ambiental, houve aumento das pressões nacionais e internacionais para a utilização sustentada das florestas tropicais, promovendo melhoria de técnicas visando compatibilizar o desenvolvimento ambiental, social e econômico, através do manejo florestal sustentável.

Porém, segundo Acselrad (1995), para dizer se uma determinada prática florestal é sustentável, é preciso recorrer a uma comparação de atributos entre dois momentos distintos: entre passado e presente e entre presente e futuro. Como as poucas áreas manejadas ainda encontram-se no primeiro ciclo, há 
dificuldades de se determinar se as técnicas atualmente desenvolvidas conduzem à sustentabilidade do manejo florestal.

Diversas formulações teóricas têm buscado abordar as relações entre economia, sociedade e ambiente, buscando incluir a problemática ambiental no âmbito das análises econômicas. As questões atuais relativas às possibilidades do manejo florestal sustentável incorporaram novas variáveis, a partir das contribuições de diversas áreas de conhecimento, até então ignoradas ou tratadas superficialmente nas formulações sobre o manejo florestal (SOUZA, 2002).

Entretanto, as pesquisas desenvolvidas para comprovar a viabilidade do manejo florestal madeireiro têm privilegiado principalmente a verificação de aspectos técnico-científicos. As análises econômicas ainda estão limitadas a experiências em projetos demonstrativos, carecendo de informações sobre o comportamento econômico em escala empresarial e envolvendo mais de um ciclo de corte, que é o período decorrido entre dois momentos de exploração na mesma área (SOUZA, 2002).

Apesar da atividade do manejo florestal sustentado requerer um longo período para a obtenção de produtos, o seu planejamento pode ser realizado sob a ótica do curto prazo. A diferença qualitativa essencial entre o curto prazo e o longo prazo, é que este é o planejamento para mudanças, enquanto aquele é a adaptação às mudanças. Nesse sentido, percebe-se que a capacidade de produção da empresa em função da área é fixa, tornando a sua liberdade de ação limitada.

A análise do ponto de equilíbrio é uma análise dos custos e das receitas em função do nível de atividade. $\mathrm{O}$ ponto de equilíbrio monetário demonstra o quanto é necessário faturar por ano para cobrir todos os custos da atividade de manejo, sem ganhos e perdas, e o ponto de equilíbrio de área indica qual a área anual mínima necessária para ser trabalhada, de forma que as receitas auferidas pelas vendas das toras se igualem aos custos de produção (TIMOFEICZYK JÚNIOR, 2004).

Entre os custos envolvidos no processo de produção de madeira, estão aqueles cuja avaliação técnico-econômica é de grande importância, como é o caso do custo de oportunidade da terra, já que esse recurso produtivo representa um alto investimento e é de relativa permanência. A quantificação do custo de oportunidade pelo uso da terra, que é a remuneração periódica e definitiva do montante investido em terra, deve considerar o juro sobre o capital investido nesse recurso de produção, e não o seu valor total (BERGER, 1985). De acordo com Timofeiczyk Júnior et al. (2007), a consideração do custo de oportunidade da terra no manejo de florestas tropicais altera a estrutura de custo de produção, causando grande impacto no custo fixo.

Dentro desse contexto, o objetivo do presente trabalho foi determinar o ponto de equilíbrio monetário e de área em escala empresarial do manejo florestal de baixo impacto envolvendo dois ciclos de corte, e analisar o impacto do custo de oportunidade da terra no ponto de equilíbrio.

\section{MATERIAL E MÉTODOS}

\section{Área de estudo}

A descrição da floresta que gerou os dados para a realização desse trabalho foi fornecida pelo Projeto de Apoio ao Manejo Florestal Sustentável na Amazônia-MMA/IBAMA/PPG7, Projeto HuaiaMissu. A floresta está localizada no município de Marcelândia, no Estado do Mato Grosso, a $873 \mathrm{~km}$ de Cuiabá.

A área total da fazenda é de 145.000 ha, e a Área de Manejo Florestal (AMF) é de 116.348 ha. Cada Unidade de Produção Anual (UPA), que é a área a ser explorada anualmente, tem 4.653,92 ha. A floresta produz toras de madeira tropical, e o principal destino da produção são as indústrias de laminados e serrados dos municípios de Sinop e Marcelândia, no Mato Grosso.

\section{Fonte de dados primários}

A empresa responsável pela execução do manejo florestal e pela comercialização das toras forneceu os dados de todos os recursos físicos e financeiros necessários para a execução do manejo florestal, assim como as receitas auferidas pela venda da produção.

\section{Identificação das atividades e operações}

Foram considerados dois ciclos de corte de 25 anos cada um, com base na Instrução Normativa 04 do Ministério do Meio Ambiente (MMA), que regulamenta o manejo florestal em escala empresarial 
na Amazônia. Na figura 1 está representada a metodologia adotada, onde cada quadrado representa uma UPA, do total de 25 UPAs. O primeiro ciclo de corte pode ser considerado como implantação do sistema de manejo florestal, onde são exploradas as UPAS de número 1 a 25 . Ao iniciar o segundo ciclo de corte, cujo sistema de manejo florestal já está implantado, retorna-se à primeira UPA, representada pela simbologia 1s nessa figura. Esse procedimento repete-se ao longo dos anos.

Figura 1. Unidades de produção anual (UPA) nos dois ciclos de produção.

Figure 1. Annual production units in the two cutting cicles.

\begin{tabular}{|c|c|c|c|c|c|c|c|c|c|}
\hline 1 & 2 & 3 & 4 & 5 & 6 & 7 & 8 & 9 & 10 \\
\hline 11 & 12 & 13 & 14 & 15 & 16 & 17 & 18 & 19 & 20 \\
\hline 21 & 22 & 23 & 24 & 25 & $1 \mathrm{~s}$ & $2 \mathrm{~s}$ & $3 \mathrm{~s}$ & $4 \mathrm{~s}$ & $5 \mathrm{~s}$ \\
\hline $6 \mathrm{~s}$ & $7 \mathrm{~s}$ & $8 \mathrm{~s}$ & $9 \mathrm{~s}$ & $10 \mathrm{~s}$ & $11 \mathrm{~s}$ & $12 \mathrm{~s}$ & $13 \mathrm{~s}$ & $14 \mathrm{~s}$ & $15 \mathrm{~s}$ \\
\hline $16 \mathrm{~s}$ & $17 \mathrm{~s}$ & $18 \mathrm{~s}$ & $19 \mathrm{~s}$ & $20 \mathrm{~s}$ & $21 \mathrm{~s}$ & $22 \mathrm{~s}$ & $23 \mathrm{~s}$ & $24 \mathrm{~s}$ & $25 \mathrm{~s}$ \\
\hline
\end{tabular}

\section{Custos fixos}

Os custos fixos incluem todas as formas de remunerações decorrentes da manutenção dos recursos fixos. Os componentes dos custos fixos são:

a) Imposto Territorial Rural - ITR;

b) Depreciação;

c) Juros sobre o Capital Próprio;

d) Custos Administrativos;

e) Custo de Oportunidade da Terra.

Para o cálculo da depreciação, optou-se pelo critério de depreciação linear, largamente utilizado na literatura econômica. Conforme Hildebrand (1995), a depreciação linear é calculada por meio da seguinte fórmula:

em que:

$$
\text { De }=(\text { V.A. }- \text { V.R. }) / \text { V.U }
$$

$\mathrm{De}=$ depreciação $(\mathrm{R} \$ / \mathrm{ano})$;

$\mathrm{V} . \mathrm{A}=$ valor de aquisição $(\mathrm{R} \$)$;

$\mathrm{V} . \mathrm{R}=$ valor residual $(\mathrm{R} \$)$;

$\mathrm{V} . \mathrm{U}=$ vida útil (anos).

A remuneração do capital próprio representa o valor perdido pela não aplicação na melhor alternativa econômica (HILDEBRAND, 1995). Neste estudo, a taxa de juros utilizada para a determinação desse custo foi de $12 \%$ ao ano, com base no trabalho de Timofeiczyk Júnior (2004). De acordo com Hildebrand (1995), a fórmula para calcular a remuneração do capital próprio é:

em que:

$$
R C=V A .\left((1+i)^{n} \cdot i\right) /\left((1+i)^{n}-1\right)-D
$$

$\mathrm{RC}=$ remuneração do capital $(\mathrm{R} \$)$;

$\mathrm{VA}=$ valor presente da aplicação ou aquisição $(\mathrm{R} \$) ;$

$\mathrm{i}=$ taxa de juros, expressa em decimal $(0,0 \mathrm{i})$;

$\mathrm{D}=$ depreciação anual $(\mathrm{R} \$)$;

$\mathrm{n}=$ tempo (anos).

A taxa de remuneração adotada como custo de oportunidade da terra foi de $3 \%$ ao ano. Esse valor é recomendado por Turra (1990), que afirma não haver justificativa para o uso de outros valores, baseado em estudos econômicos da agricultura brasileira, uma vez que a terra é um ativo de baixa liquidez, ou seja, não é um bem que rapidamente possa ser convertido em aplicações mais rentáveis. De acordo com Rezende et al. (1994), a função do custo de oportunidade da terra pode ser dada por: 
em que:

$\mathrm{CT}=$ custo de oportunidade da terra $(\mathrm{R} \$ / \mathrm{ha})$;

$\mathrm{VC}=$ valor de mercado da terra, por hectare;

$\mathrm{TX}=$ taxa anual de remuneração da terra $(\%)$.

\section{Custos variáveis}

Para a execução do Plano de Manejo Florestal Sustentável (PMFS), é necessária a elaboração do Plano Operacional Anual (POA), que deverá conter todas as atividades florestais da empresa num determinado ano, tais como:

a) Atividade pré-exploratória, composta pelas seguintes operações:

Elaboração do Plano de Manejo Florestal Sustentado - PMFS;

- Confecção de mapas e inventário amostral.

Censo florestal;

- Demarcação do talhão, aberturas de picadas, avaliação e mapeamento das árvores.

Corte de cipós e taxas do Ibama (pré-exploratória).

b) Atividade exploratória, composta pelas seguintes operações:

Taxas do Ibama;

Operações de exploração;

- Demarcação e abertura de estradas secundárias e esplanadas, derrubada, arraste, traçamento, cubagem e carregamento das toras nos caminhões.

c) Atividade pós-exploratória, composta pelas seguintes operações:

Tratos silviculturais;

- Corte de cipós e favorecimento das espécies remanescentes.

Limpeza de estradas secundárias e esplanadas;

Proteção florestal, monitoramento e desenvolvimento da floresta.

d) Impostos.

\section{Levantamento das receitas}

Foram utilizados os relatórios mensais de vendas da empresa, contendo as espécies comercializadas e o preço de comercialização das toras. Amaral et al. (1998) afirmam que o acréscimo de volume a ser explorado no segundo ciclo é de $16 \%$, em razão dos tratos silviculturais. A produção atual dessa floresta é de $12 \mathrm{~m}^{3} /$ ha embarcados, porém a empresa responsável pela execução do manejo da referida floresta tem uma expectativa de que o volume aumente para $15 \mathrm{~m}^{3} /$ ha no segundo ciclo, em função da implementação dos tratos silviculturais.

\section{Ponto de equilíbrio}

A análise do ponto de equilíbrio é uma análise dos custos e das receitas em função do nível de atividade (FIGUEIREDO; CAGGIANO, 1997). O ponto de equilíbrio é importante para a decisão de planejamento que necessita ser tomada, e a determinação desse ponto traz considerações quanto ao possível risco de certas alternativas. Os autores enfatizam que essa análise é o centro do planejamento do lucro de curto prazo, tendo aplicação em grande número de atividades.

Conforme Timofeiczyk Júnior (2004), o ponto de equilíbrio de área indica qual a área anual mínima necessária para ser trabalhada, de forma que as receitas auferidas pelas vendas se igualem aos custos de produção. Nesse nível de produção, a empresa consegue absorver todos os seus custos fixos. De acordo com Figueiredo; Caggiano (1997), a fórmula para o cálculo do ponto de equilíbrio é:

$$
\mathrm{PE}=\mathrm{CFT} / \mathrm{MCU}
$$

em que:

$\mathrm{PE}=$ ponto de equilíbrio (ha/ano);

$\mathrm{CFT}=$ custo fixo total $(\mathrm{R} \$ / \mathrm{ano})$;

$\mathrm{MCU}=$ margem de contribuição unitária (R\$/ha). 
Segundo Figueiredo; Caggiano (1997), a margem de contribuição unitária (R \$ha) é a diferença entre a receita e os custos variáveis por hectare, sendo dada pela seguinte fórmula:

$$
\mathrm{MCU}=\mathrm{R}(\mathrm{R} \$ / \mathrm{ha})-\mathrm{CV}(\mathrm{R} \$ / \mathrm{ha})
$$

em que:

$\mathrm{MCU}=$ margem de contribuição unitária $(\mathrm{R} \$ / \mathrm{ha})$;

$\mathrm{R}(\mathrm{ha})=$ receita por hectare $(\mathrm{R} \$ / \mathrm{ha})$;

$\mathrm{CV}=$ custo variável por hectare $(\mathrm{R} \$ / \mathrm{ha})$.

Horngren (1985) afirma que o ponto de equilíbrio monetário demonstra o quanto é necessário faturar por ano para cobrir todas as despesas, sem ganhos e perdas, sendo dado pela seguinte fórmula:

em que:

$\mathrm{PEM}=\mathrm{CFT} /(\mathrm{MCU} / \mathrm{R}(\mathrm{ha}))$

$\mathrm{PEM}=$ ponto de equilíbrio monetário (R\$/ano);

$\mathrm{CFT}=$ custo fixo total $(\mathrm{R} \$ / \mathrm{ano})$;

$\mathrm{MCU}=$ margem de contribuição unitária $(\mathrm{R} \$ / \mathrm{ha})$;

$\mathrm{R}(\mathrm{ha})=$ receita por hectare $(\mathrm{R} \$ / \mathrm{ha})$.

\section{RESULTADOS E DISCUSSÕES}

Quando da realização deste estudo, a empresa estava fazendo a primeira intervenção nas Unidades de Produção Anual (UPA). No atual nível de operação e comercialização, que é de $12 \mathrm{~m}^{3} / \mathrm{ha}$ distribuídos em várias espécies, verificou-se que o custo fixo permaneceu inalterado no primeiro ciclo, porém os custos médios de produção aumentaram independentemente da consideração ou não do custo de oportunidade da terra. Sem considerar o custo da terra, o custo médio de produção aumentou de R\$ $62,18 / \mathrm{m}^{3}$ no início do primeiro ciclo para $\mathrm{R} \$ 65,71 / \mathrm{m}^{3}$ ao final dele. Ao considerar esse custo, ocorreu um aumento de $\mathrm{R} \$ 85,55$ para $\mathrm{R} \$ 89,08$ ao longo do primeiro ciclo. A elevação do custo médio de produção ocorreu em função da implantação da atividade pós-exploratória nas Unidades de Produção Anual (UPA), ocasionando aumento nos custos variáveis.

Com o aumento da produção de 12 para $15 \mathrm{~m}^{3} / \mathrm{ha}$ no segundo ciclo, houve redução do custo fixo médio nas duas situações analisadas, já que o aumento da produção diluiu o custo fixo numa quantidade maior de toras de madeira. No segundo ciclo de corte, algumas operações da atividade exploratória, como a demarcação e abertura de estradas secundárias e esplanadas não são executadas, em função da limpeza de estradas secundárias e esplanadas que foram realizadas a partir do primeiro ciclo, com intervalo de cinco anos. Das operações da atividade pré-exploratória, o inventário amostral não foi executado, em razão da medição das parcelas permanentes. A demarcação de talhões também não foi realizada no segundo ciclo por já ter sido implementada no primeiro ciclo. A redução de operações no cronograma operacional, aliada ao aumento da produção, ocasionou redução nos custos médios de produção para $\mathrm{R} \$$ 50,32 sem custo de oportunidade da terra, e para $\mathrm{R} \$ 66,59$ considerando esse custo (Tabela 1).

Tabela 1. Custos médios de produção $\left(\mathrm{R} \$ / \mathrm{m}^{3}\right)$ com e sem custo de oportunidade da terra.

Table 1. Average production costs $\left(\mathrm{R} \$ / \mathrm{m}^{3}\right)$ with and without land opportunity.

\begin{tabular}{lccc}
\hline \multirow{2}{*}{ Sem custo de oportunidade da terra } & \multicolumn{3}{c}{ Ciclos de corte } \\
\cline { 2 - 4 } & Início do 1 $\mathbf{1}^{\mathbf{0}}$ ciclo & Final do 1 $\mathbf{1}^{\mathbf{0}}$ ciclo & $\mathbf{2}^{\mathbf{0}}$ ciclo \\
\hline Custo fixo médio & 22,62 & 22,62 & 18,10 \\
Custo variável médio & 39,56 & 43,09 & 32,22 \\
Custo total médio & 62,18 & 65,71 & 50,32 \\
\hline \multirow{2}{*}{ Com custo de oportunidade da terra } & \multicolumn{3}{c}{ Ciclos de corte } \\
\cline { 2 - 4 } Custo fixo médio & Início do 1 $\mathbf{1}^{\mathbf{0}}$ ciclo & Final do 1 $\mathbf{1}^{\mathbf{0}}$ ciclo & $\mathbf{2}^{\mathbf{0}}$ ciclo \\
Custo variável médio & 45,99 & 45,99 & 34,37 \\
Custo total médio & 39,56 & 43,09 & 32,22 \\
\hline
\end{tabular}

Fonte: Timofeiczyk Júnior et al. ( 2007). 
A elevação no custo médio de produção e consequentemente nos custos totais ao longo do primeiro ciclo aumentaram o tamanho mínimo anual das UPA para que ocorra igualdade entre receitas e custos, como é possível verificar na tabela 2. A atribuição do custo de oportunidade da terra na determinação do tamanho das UPA elevou significativamente o tamanho de área necessária para que houvesse equilíbrio entre receitas e custos. Considerando o custo de oportunidade da terra, houve um grande déficit de área para ser explorada. No início do primeiro ciclo, esse déficit foi de 3.027 ha, ou seja, o tamanho da UPA teria que ser 65\% maior para que ocorresse igualdade entre receitas e despesas. Com a implementação da atividade pós-exploratória no primeiro ciclo, houve aumento dos custos variáveis e consequentemente dos custos totais, elevando o déficit para $89 \%$.

A redução de operações, aliada ao aumento da produção no segundo ciclo, ocasionou redução no déficit de área mínima necessária para que os custos se igualem às receitas, passando para 210 ha. Ainda considerando o custo de oportunidade da terra, verificou-se que foi necessária uma área efetiva de 209.896 ha no primeiro ciclo, subdividida em 25 UPA, para que ocorresse sustentabilidade econômica. Como a área de efetivo manejo é de 116.348 ha, ocorreu uma defasagem de 93.548 ha. No segundo ciclo, a necessidade de área total foi de 116.500 ha, com o déficit reduzindo para 5.240 ha.

Levando-se em conta a afirmação de Graça (1997) de que pequenos produtores florestais não vêem a terra como um bem conceitualmente disponibilizável para auferir remuneração alternativa, podese considerar a não existência de um custo de oportunidade da terra quando essa é utilizada na produção de bens florestais. Desconsiderando esse custo, os tamanhos das UPA foram suficientes para cobrir os custos de produção e ainda gerar lucro (Tabela 2). No início do primeiro ciclo, é necessário que sejam explorados 3.778 ha para que as receitas das vendas das toras possam cobrir os custos de produção. Como o tamanho das UPA é de 4.654 ha, houve um superávit de área de 876 ha.

Entretanto, para que os custos sejam cobertos pelas receitas, a área a ser explorada aumentou em determinados intervalos de tempo. Ao final do primeiro ciclo, o superávit de área reduziu para 328 ha, porém o suficiente ainda para gerar uma determinada margem de lucro. Ao iniciar o segundo ciclo, a área necessária para cobrir os custos de produção foi reduzida para 2.392 ha. Em relação ao ponto de equilíbrio, as UPA apresentaram um excedente de $49,2 \%$, aumentando a margem de lucro a partir do segundo ciclo (Tabela 2).

Tabela 2. Ponto de equilíbrio de área com e sem custo de oportunidade da terra (ha).

Table 2. Break-even-point of area with and without the opportunity cost of the land (ha).

\begin{tabular}{lccccc}
\hline \multirow{2}{*}{ Ano } & \multirow{2}{*}{ UPA } & \multicolumn{2}{c}{ Com custo da terra } & \multicolumn{2}{c}{ Sem custo da terra } \\
\cline { 3 - 6 } & & Área equilíbrio & Diferença & Área equilíbrio & Diferença \\
\hline 1 & 4.654 & 7.681 & -3.027 & 3.778 & 876 \\
2 & 4.654 & 7.883 & -3.229 & 3.877 & 777 \\
5 & 4.654 & 8.094 & -3.440 & 3.981 & 673 \\
6 & 4.654 & 8.259 & -3.606 & 4.062 & 592 \\
11 & 4.654 & 8.430 & -3.776 & 4.146 & 508 \\
16 & 4.654 & 8.609 & -3.955 & 4.234 & 420 \\
21 & 4.654 & 8.796 & -4.142 & 4.326 & 328 \\
26 & 4.654 & 4.864 & -210 & 2.392 & 2.262 \\
31 & 4.654 & 4.864 & -210 & 2.392 & 2.262 \\
41 & 4.654 & 4.864 & -210 & 2.392 & 2.262 \\
51 & 4.654 & 4.864 & -210 & 2.392 & 2.262 \\
\hline
\end{tabular}

Fonte: Timofeiczyk Júnior et al. (2007).

Não considerando o custo da terra, é necessária uma área efetiva de 103.245 ha no primeiro ciclo, subdividida em 25 UPA, para que ocorra sustentabilidade econômica. Nesse caso, ocorreu um superávit de 13.103 ha. No segundo ciclo, o superávit de área aumentou para 56.550 ha.

Analisando o ponto de equilíbrio sob a ótica monetária, considerando o custo de oportunidade da terra, houve uma grande diferença negativa entre a receita obtida e a receita de equilíbrio. No início do primeiro ciclo, o déficit foi de $65 \%$ (-R \$ 2.449.292), aumentando para $89 \%$ (-R \$ 3.351.482) ao final dele (Tabela 3). 
No segundo ciclo, em função da redução dos custos totais e do aumento da produção, o déficit entre a receita realizada e a de equilíbrio diminuiu para - $\mathrm{R} \$ 212.062$ (Tabela 3). Ao se desconsiderar o custo de oportunidade da terra, constatou-se que a receita total realizada pela empresa situou-se acima do ponto de lucro zero, justificada pela área trabalhada anualmente. Entretanto, essa diferença positiva, que no início do primeiro ciclo foi de $\mathrm{R} \$ 708.517$, diminuiu para $\mathrm{R} \$ 264.732$ ao final desse. Já no segundo ciclo, em função da redução dos custos totais e do aumento da produção, elevou-se a diferença entre a receita monetária de equilíbrio e a realizada, aumentando a margem de lucro (Tabela 3).

Tabela 3. Ponto de equilíbrio monetário com e sem custo de oportunidade da terra (em R\$). Table 3. Monetary break-even-point with and without the opportunity cost of the land (R\$).

\begin{tabular}{lccccc}
\hline \multirow{2}{*}{ Ano } & \multirow{2}{*}{ Receita total } & \multicolumn{2}{c}{ Com custo da terra } & \multicolumn{2}{c}{ Sem custo da terra } \\
\cline { 3 - 6 } & & Receita equilíbrio & Diferença & Receita equilíbrio & Diferença \\
\hline 1 & 3.765 .487 & 6.214 .779 & -2.449 .292 & 3.056 .970 & 708.517 \\
2 & 3.765 .487 & 6.377 .897 & -2.612 .410 & 3.137 .207 & 628.280 \\
5 & 3.765 .487 & 6.548 .746 & -2.783 .259 & 3.221 .247 & 544.240 \\
6 & 3.765 .487 & 6.682 .122 & -2.916 .635 & 3.286 .854 & 478.633 \\
11 & 3.765 .487 & 6.821 .044 & -3.055 .557 & 3.355 .190 & 410.297 \\
16 & 3.765 .487 & 6.965 .865 & -3.200 .378 & 3.426 .427 & 339.060 \\
21 & 3.765 .487 & 7.116 .969 & -3.351 .482 & 3.500 .755 & 264.732 \\
26 & 4.706 .858 & 4.918 .920 & -212.062 & 2.419 .506 & 2.287 .352 \\
31 & 4.706 .858 & 4.918 .920 & -212.062 & 2.419 .506 & 2.287 .352 \\
41 & 4.706 .858 & 4.918 .920 & -212.062 & 2.419 .506 & 2.287 .352 \\
51 & 4.706 .858 & 4.918 .920 & -212.062 & 2.419 .506 & 2.287 .352 \\
\hline
\end{tabular}

Fonte: Timofeiczyk Júnior et al (2007).

\section{CONCLUSÕES}

- Sem considerar o custo de oportunidade da terra, o custo variável médio apresentou-se como o principal componente do custo médio, elevando-se ao longo do primeiro ciclo de produção. Essa elevação ocorre em função da realização da atividade pós-exploratória.

- A consideração do custo de oportunidade da terra altera a estrutura de custos do manejo, com o custo fixo médio tornando-se maior que o custo variável médio.

- O custo fixo no primeiro ciclo ficou inalterado monetariamente. No segundo ciclo, o aumento da produção diluiu o custo fixo por uma quantidade maior de produto, ocasionando a redução do custo fixo médio.

- O ponto de equilíbrio de área e monetário demonstrou que a execução do manejo florestal em florestas tropicais requer uma extensa área de terra para que ocorra sustentabilidade econômica ao longo do tempo.

- Ao incluir o custo de oportunidade da terra na estrutura de custos, verificou-se um incremento significativo no tamanho da área necessária para que haja igualdade entre receitas e custos.

- A implementação da atividade pós-exploratória no primeiro ciclo acarretou no aumento do ponto de equilíbrio monetário e de área. No segundo ciclo, ocorreu redução no ponto de equilíbrio de área e monetário.

\section{REFERÊNCIAS}

ACSELRAD, H. Sustentabilidade, discursos e disputas. In: WORKSHOP SUSTENTABILIDADE: Perspectivas Não-Governamentais, 1995, Rio de Janeiro. Anais... Rio de Janeiro: IBASE, 1995.

AMARAL, P.; VERÍSSIMO, A.; BARRETO, P.; VIDAL, E. Floresta para sempre: um manual para a produção de madeira na Amazônia. Belém: Imazon, 1998. 130p.

BERGER, R. Aplicação de critérios econômicos para determinação da maturidade financeira de povoamentos de eucaliptos. 73 f. Tese (Professor titular) - Departamento de Economia Rural e Extensão, Setor de Ciências Agrárias, Universidade Federal do Paraná, Curitiba, 1985, 
FIGUEIREDO, S.; CAGGIANO, P.C. Controladoria-teoria e prática. 2. ed. São Paulo: Atlas, 1997.

GRAÇA, L. R. Elementos de custos de produção florestal: apropriações a análises. Curitiba: Fupef Silviconsult Engenharia, 1997. p. 1-21.

HIGUCHI, N. Utilização e manejo de recursos madeireiros das florestas tropicais úmidas. Acta Amazônica, Manaus, v. 204, n. 3/4, p. 275-278, 1994.

HILDEBRAND, E. Sistema de apropriação e análise de custos para a empresa florestal. 145 f. Dissertação (Mestrado em Ciências Florestais) - Setor de Ciências Agrárias, Universidade Federal do Paraná, Curitiba, 1995.

HORNGREN, C. T. Introdução à contabilidade gerencial. 5. ed. Rio de Janeiro: Prentice-hall do Brasil, 1985.

REZENDE, J. L. P.; LOPES, H. V. S.; NEVES, A. R.; PAULA JUNIOR, G. G. A importância do custo da terra na determinação da idade ótima de corte de povoamentos de Eucalyptus. Revista Árvore, Viçosa, MG, v. 18, n. 1, p. 45-55, 1994.

SOUZA, A. L. P. Desenvolvimento sustentável, manejo florestal e o uso dos recursos madeireiros na Amazônia: desafios, possibilidades e limites. Belém: UFPA/NAEA, 2002. 255 p.

TIMOFEICZYK JÚNIOR, R.; BERGER, R.; SOUSA, R. A. T. M.; SILVA, V. S. M. Custo de oportunidade da terra no manejo de baixo impacto em florestas tropicais - um estudo de caso. Floresta, Curitiba, v. 37, n. 3, p. 327-338, 2007.

TIMOFEICZYK JÚNIOR, R. Análise Econômica do Manejo de Baixo Impacto em Florestas Tropicais - Um Estudo de Caso. 126 f. Tese (Doutorado em Engenharia Florestal) - Setor de Ciências Agrárias, Universidade Federal do Paraná, Curitiba, 2004.

TURRA, F. E. Análise de diferentes métodos de cálculo de custos de produção na agricultura brasileira. Curitiba: OCEPAR, 1990. 86 p. 